\title{
Corela
}

Cognition, représentation, langage

HS-1 1 | 2012

RJC Cotexte, contexte, situation

\section{La réduction vocalique dans la variété salentine d'italien}

Ilaria Libera Margherita

\section{OpenEdition}

\section{Journals}

Édition électronique

URL : http://journals.openedition.org/corela/2510

DOI : $10.4000 /$ corela. 2510

ISSN : $1638-573 \mathrm{X}$

Éditeur

Cercle linguistique du Centre et de I'Ouest - CerLICO

Référence électronique

\|laria Libera Margherita, «La réduction vocalique dans la variété salentine d'italien », Corela [En ligne], HS-11 | 2012, mis en ligne le 02 avril 2012, consulté le 30 avril 2019. URL : http:// journals.openedition.org/corela/2510 ; DOI : 10.4000/corela.2510

Ce document a été généré automatiquement le 30 avril 2019

\section{(c) (i) (2)(2)}

Corela - cognition, représentation, langage est mis à disposition selon les termes de la licence Creative Commons Attribution - Pas d'Utilisation Commerciale - Partage dans les Mêmes Conditions 4.0 International. 


\title{
La réduction vocalique dans la variété salentine d'italien
}

\author{
Ilaria Libera Margherita
}

\section{Prémisses méthodologiques}

1 Le travail s'inscrit dans une ligne de recherche sur le phénomène de la réduction vocalique dans la parole naturelle des variétés italiennes ${ }^{1}$. La réduction est un cas particulier de simplification linguistique qui concerne surtout des phénomènes phonétiques. En phonétique on parle de réduction vocalique quand les valeurs cibles des voyelles produites en isolement ne sont pas atteintes, selon le contexte. Du point de vue articulatoire c'est l'approximation des organes phonatoires à la position prévue au niveau neuromoteur, et donc la réalisation manquée de la cible. Au niveau acoustique elle entraîne des valeurs formantiques différentes. Au sens le plus large la réduction s'inscrit dans le phénomène plus général de la coarticulation, qui concerne toutes les productions vocales. La réalisation de tous les sons est reliée aux caractéristiques articulatoires du contexte phonique dans lequel ils sont insérés. Cela arrive parce que la programmation temporelle du système nerveux est simultanée à la réalisation articulatoire.

2 Le phénomène a été exploré largement, à partir de la première formulation du modèle de target undershoot par Lindblom (1963), qui reliait la réduction à la durée de la voyelle. Le modèle a été modifiée par Lindblom (1983) e Lindblom-Moon (1988), en considérant comme facteurs de coarticulation/réduction des voyelles, avec la durée, le style de parole, les stratégies communicatives des locuteurs, le débit et la structure accentuelle².

\section{Objectifs}

3 L'étude présente une recherche sur les voyelles de la variété salentine d'italien, avec une comparaison entre les systèmes vocaliques de deux sujets, un homme et une femme, et entre deux styles de parole : semi-spontanée (dans ce cas dit parole dialogique), et parole lue . L'essai phonétique tente une analyse qualitative et quantitative des phénomènes de 
réduction vocalique sur la base de la distinction, réalisée à partir de Savy \& Cutugno (1997), entre deux types de réduction:

- Réduction structurelle : reliée aux variantes accentuelles où les voyelles ont tendance à être réduites vers une forme plus ou moins centralisée, sur les deux axes, se rapprochant ainsi d'un schwa.

- Réduction non-structurelle : dûe à phénomènes de hypoarticulation, caractéristique propre de la parole spontanée. Pour évaluer les modalités de cette réduction l'étude se sert de la distinction, établie en phase d'étiquettage, entre :

- Voyelles définies : celles qui se rapprochent aux attentes phonologiques.

- Voyelles indéfinies: segments vocaliques auxquels n'est pas attribué de marque phonologique.

- Suivant Lo Prejato et al. (2004), on a opéré préliminairement, dans les systèmes atones, une distinction entre :

- Voyelles finales vraies: voyelles finales en même temps à niveau phonologique et de constituant prosodique, ou de réplique.

- Voyelles finales fausses : finales seulement à niveau phonologique, mais non dans la chaîne phonique.

\section{Matériel et méthode}

4 Le matériel audio a été enregistré (magnétophone DAT) à l'Université de Lecce dans le cadre du projet CLIPS, et il se compose de deux dialogues (20 minutes au total) de type Map task et Spot of difference, et de quelques listes des mots. Les locuteurs, un homme et une femme, sont deux étudiants universitaires de la localité qui fait l'objet de l'enquête.

Pour chaque locuteur on a décidé de considérer toutes les répétitions des vingt-deux types vocaliques identifiés :

- -Sept toniques.

- - Cinq atones.

- -Quatre finales vraies.

- -Quatre finales fausses.

Pour chaque son l'analyse acoustique se compose de :

- -Analyse spectral, au moyen du logiciel d'analyse WaveSurfer (TkDLL) ${ }^{3}$, à partir des spectrogrammes à large bande, qui a fournit la valeur des fréquences des premier (F1) et second (F2) formants, et de la fréquence fondamentalle (fo). Le mesurage a été estimé au centre de chaque réalisation.

- -Calcul des valeurs moyennes des formants de chaque voyelle et leurs écart-types.

- -Application du test $t$ - Student sur les données, pour vérifier si les résultats relatifs aux locuteurs examinés peuvent être généralisés pour l'entière population salentine. Le test a démontré que les différences des valeurs sont significatives.

- -Représentation des données au moyen des ellipses de dispersion.

Pour procéder à la comparaison entre les données des deux locuteurs, et pour mieux rendre compte des différences dues au sexe, pour les systèmes toniques et atones, on a choisi de soustraire les valeurs de fo de F1 (les résultats seront représentés sur l'axe des ordonnées), et ceux de F1 de F2 (sur l'axe des abscisses). Ces valeurs ont été converties en Bark, suivant la formule proposée par Zwicker-Ternhard.

Dans la phase d'élaboration et de représentation des données les réalisations phoniques des deux sujets ont été comparées en tenant compte du style de parole (lue/ dialogique), 
du timbre (voyelles définies/ indéfinies), du accent (voyelles toniques/ atones), de la position (voyelles finales vraies/ voyelles finales fausses).

\section{Résultats}

\section{Systèmes masculin et féminin}

\section{Figure 1}
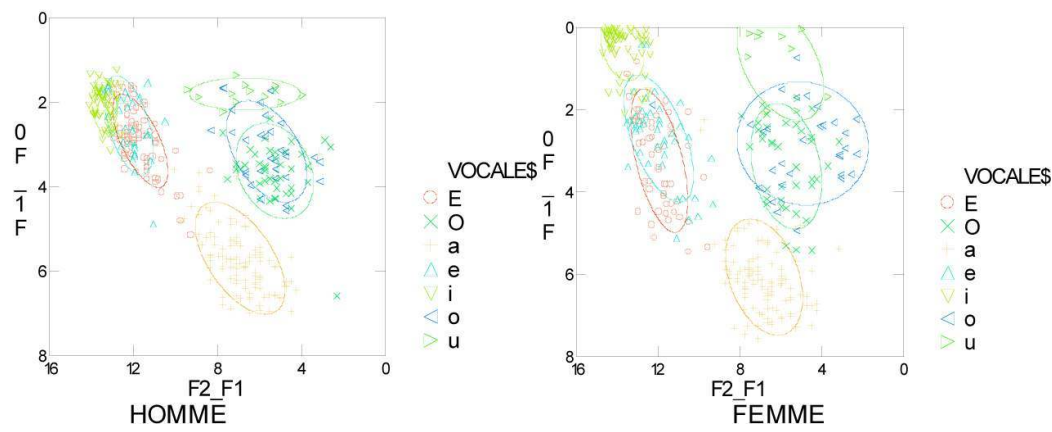

Toniques

\section{Figure 2}
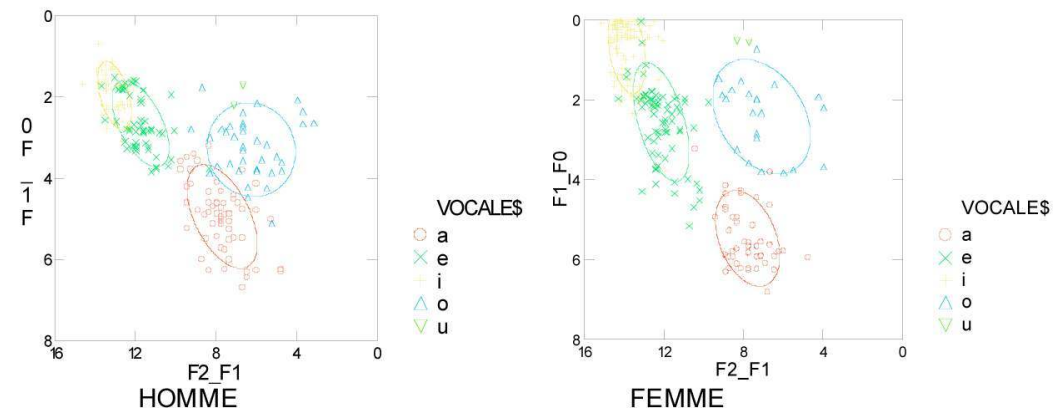

Atones

7 La comparaison des données relatives aux productions vocales des deux sujets montre la plus grande dispersion de l'espace acoustique féminin. Pour les deux séries des voyelles les réalisations féminines se situant sur une surface fréquentielle plus large.

Dans les systèmes toniques des deux locuteurs on signale la superposition des aires de dispersion des voyelles moyennes.

9 Au passage des toniques aux atones les résultats présentent une centralisation des réalisations vocaliques, plus élevée pour le locuteur masculin. 
Réduction structurelle : voyelles toniques/ atones (parole dialogique)

\section{Figure 3}
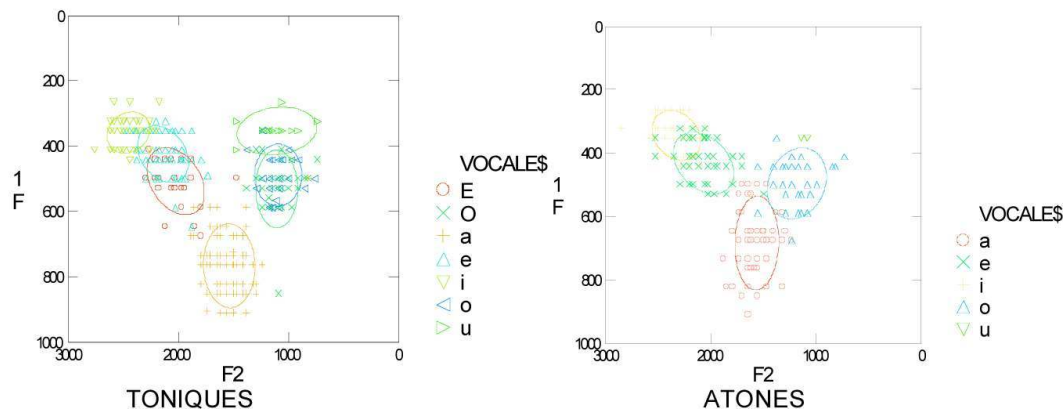

HOMME

\section{Figure 4}
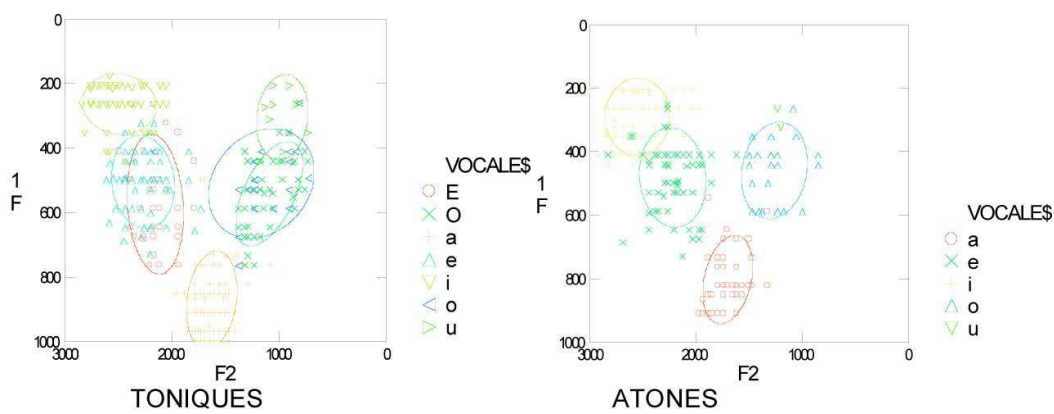

FEMME

Dans le but de mieux définir les effets de réduction reliés aux variables accentuelles et le degré de centralisation, on a calculé les moyennes des valeurs formantiques des deux systèmes vocaliques :

\begin{tabular}{|l|l|l|}
\hline & TONIQUES & ATONES \\
\hline \multirow{2}{*}{ HOMME } & F1[a] - F1 $[\mathrm{u}, \mathrm{i}]=411,83$ & $\mathrm{~F} 1[\mathrm{a}]-\mathrm{F} 1[\mathrm{u}, \mathrm{i}]=266$ \\
\cline { 2 - 3 } & $\mathrm{F} 2[\mathrm{i}]-\mathrm{F} 2[\mathrm{u}]=1372$ & $\mathrm{~F} 2[\mathrm{i}]-\mathrm{F} 2[\mathrm{u}]=1209$ \\
\hline \multirow{2}{*}{ FEMME } & $\mathrm{F} 1[\mathrm{a}]-\mathrm{F} 1[\mathrm{u}, \mathrm{i}]=601$ & $\mathrm{~F} 1[\mathrm{a}]-\mathrm{F} 1[\mathrm{u}, \mathrm{i}]=510$ \\
\cline { 2 - 3 } & $\mathrm{F} 2[\mathrm{i}]-\mathrm{F} 2[\mathrm{u}]=1530$ & $\mathrm{~F} 2[\mathrm{i}]-\mathrm{F} 2[\mathrm{u}]=1294$ \\
\hline
\end{tabular}

11 Pour les deux sujets on relève un rapport entre les dimensions de la surface acoustique et le type accentuel: l'espace fréquentiel résulte plus condensé dans les systèmes atones, dans la même mesure pour les deux formants dans les productions masculins, alors que pour la femme c'est la dimension de F2 a être plus réduite. 
Styles de parole : dialogique/lue 4

\section{Figure 5}
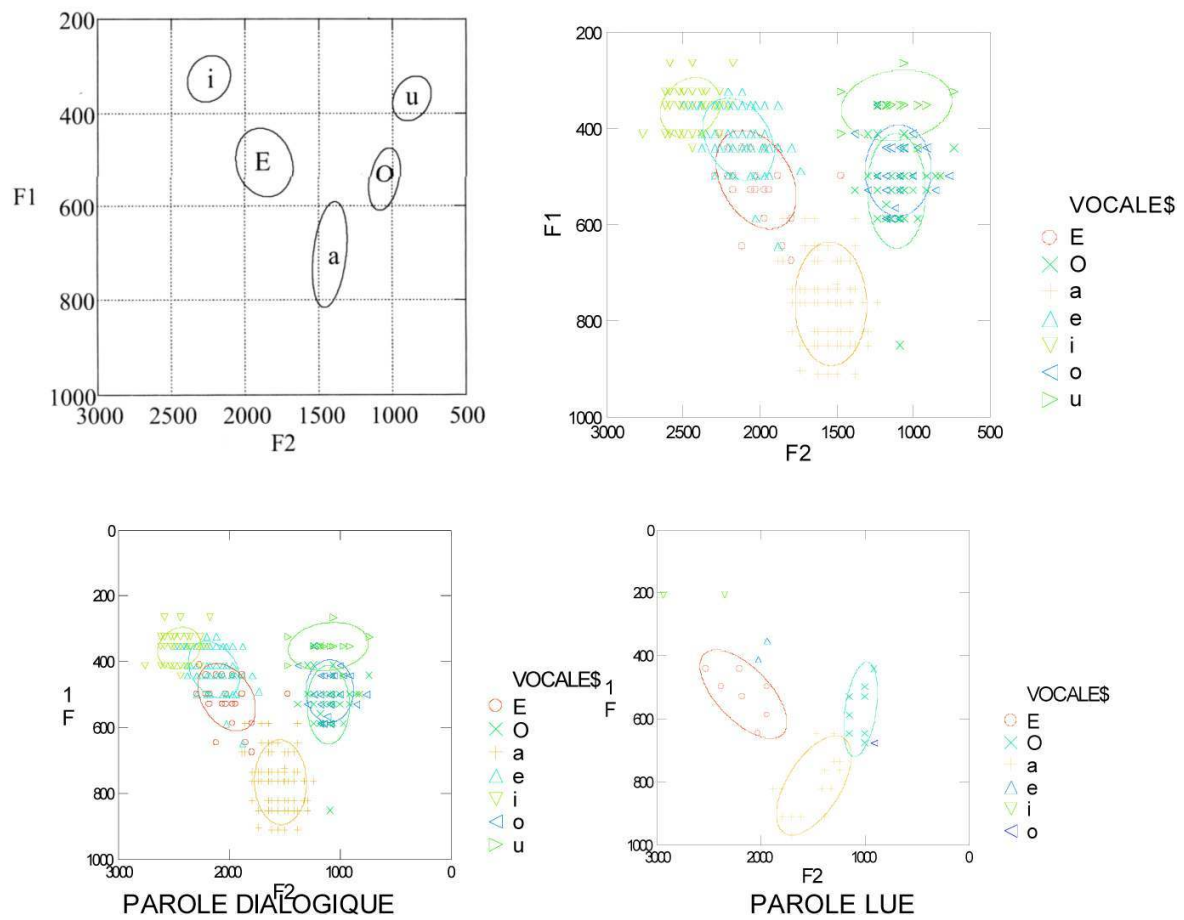

Toniques

HOMME

\section{Figure 6}

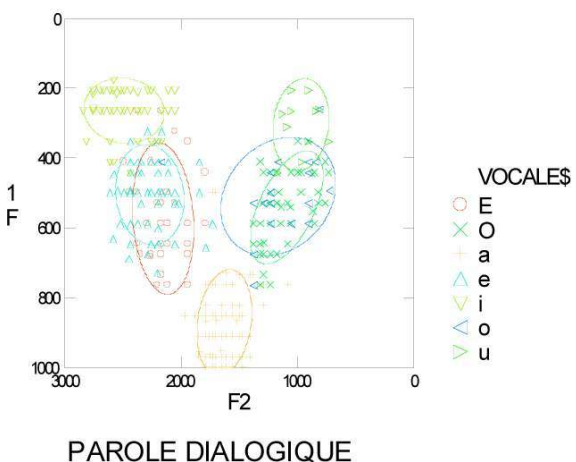

PAROLE DIALOGIQUE

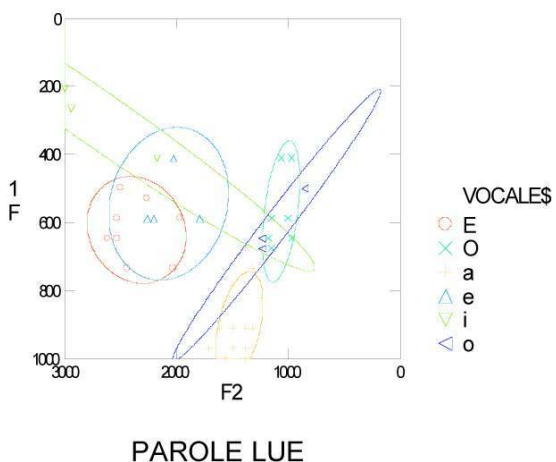

Toniques FEMME 


\section{Figure 7}
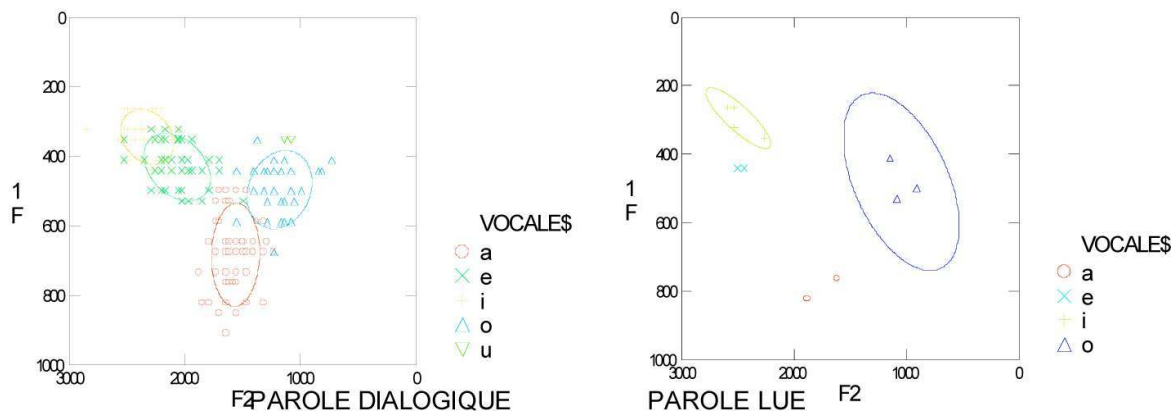

Atones

HOMME

\section{Figure 8}
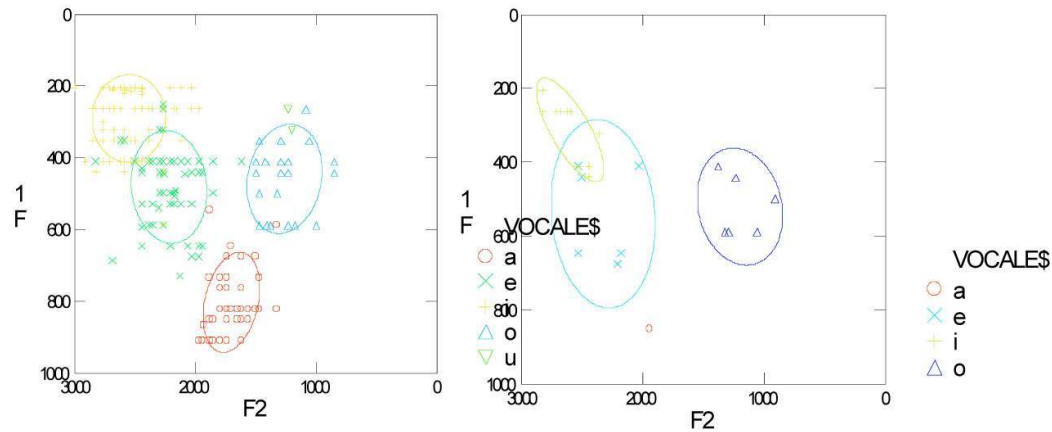

PAROLE DIALOGIQUE

PAROLE LUE

Atones

FEMME

\section{Figure 9}

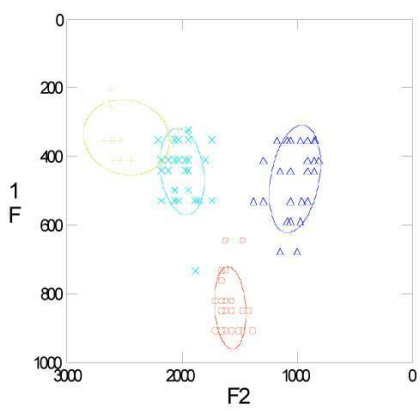

PAROLE DIALOGIQUE

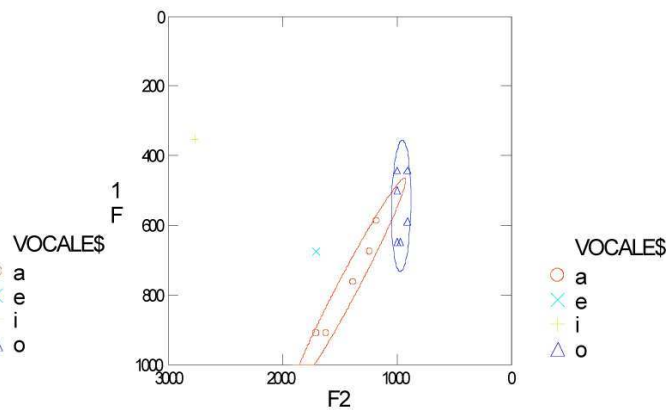

PAROLE LUE

Finales vraies

HOMME 


\section{Figure 10}

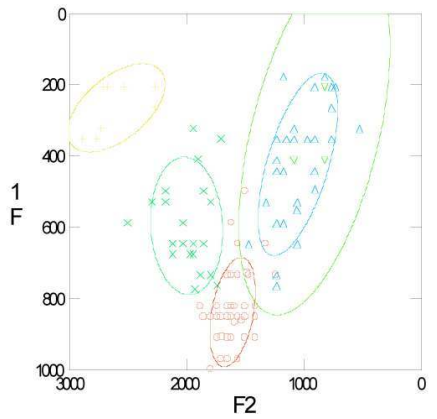

PAROLE DIALOGIQUE

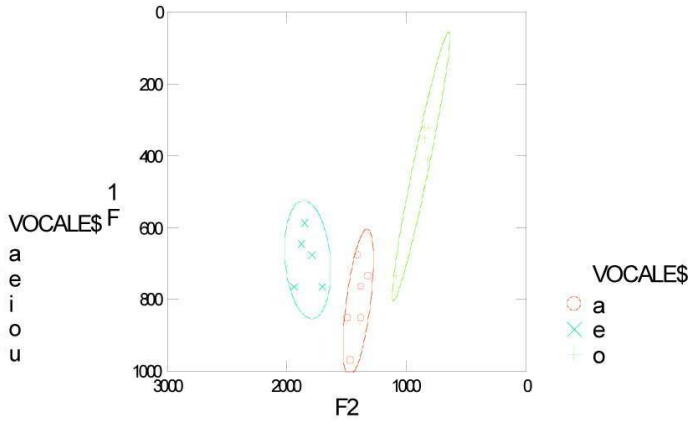

PAROLE LUE

Finales vraies

FEMME

\section{Figure 11}
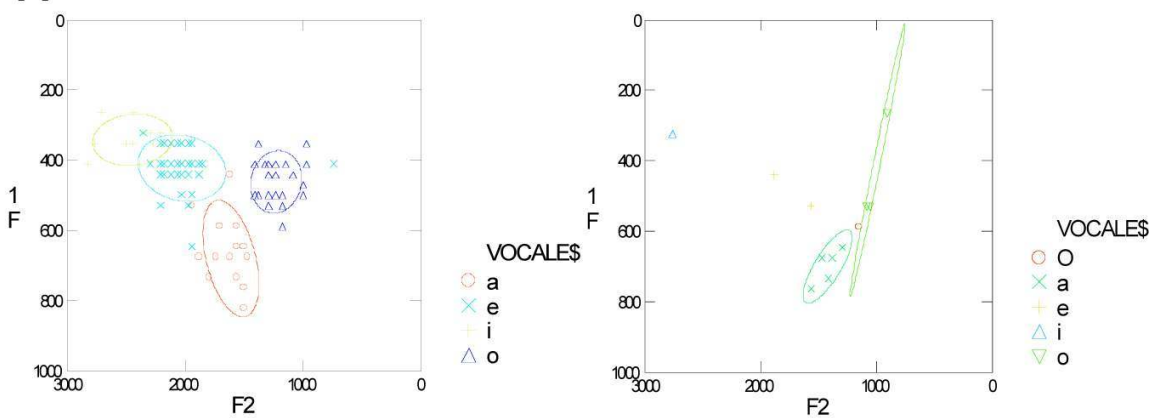

PAROLE DIALOGIQUE

PAROLE LUE

Finales fausses

HOMME

\section{Figure 12}
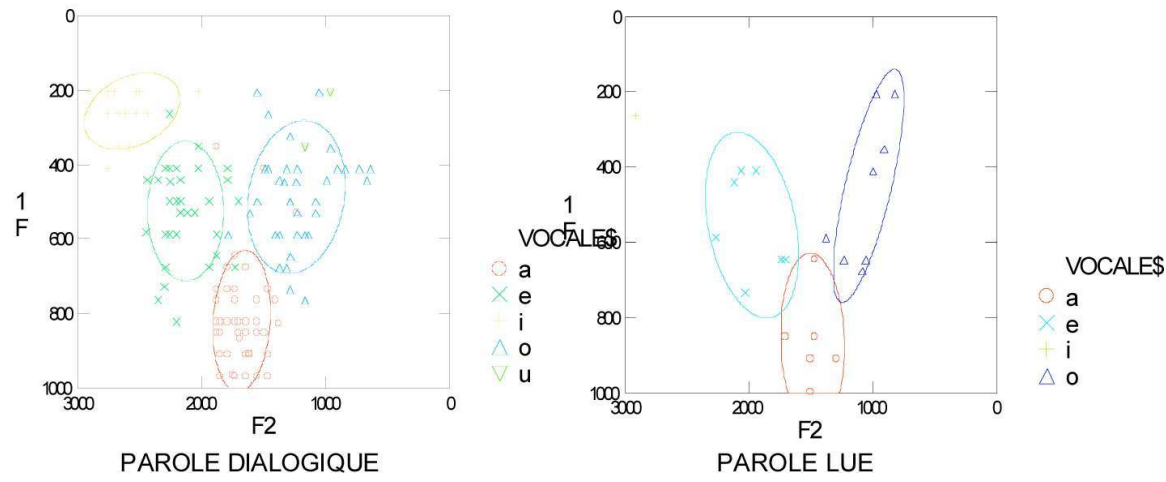

Finales fausses

FEMME

12

Les graphs montrent que la différence de style comporte un déplacement vers le centre de l'aire acoustique et un nuage des réalisations plus étendu.

13 Le style de parole semble influer surtout dans les systèmes atones, en déterminant une tendance à la réduction plus élevée. 
Réduction non structurelle : voyelles définies/ indéfinies (parole dialogique)

\section{Figure 13}
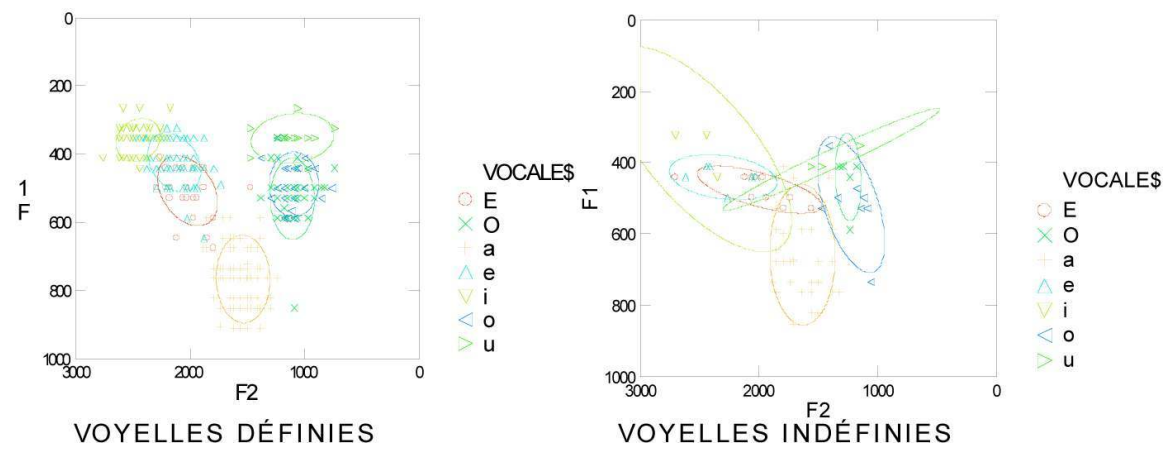

Toniques

HOMME

\section{Figure 14}
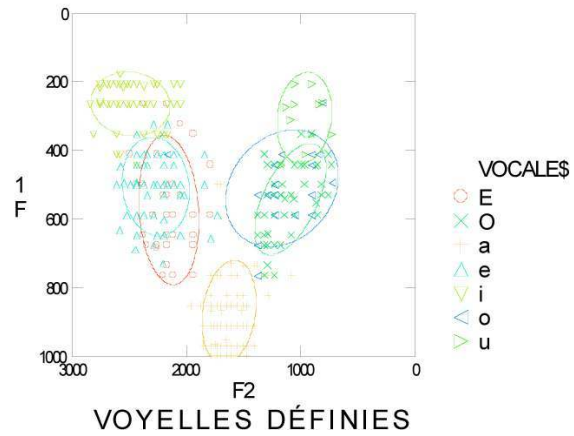

Toniques

FEMME

Figure 15

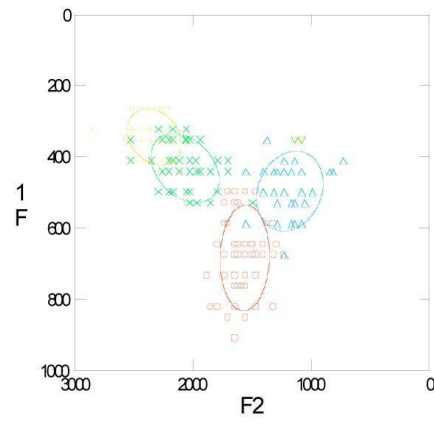

VOYELLES DÉFINIES

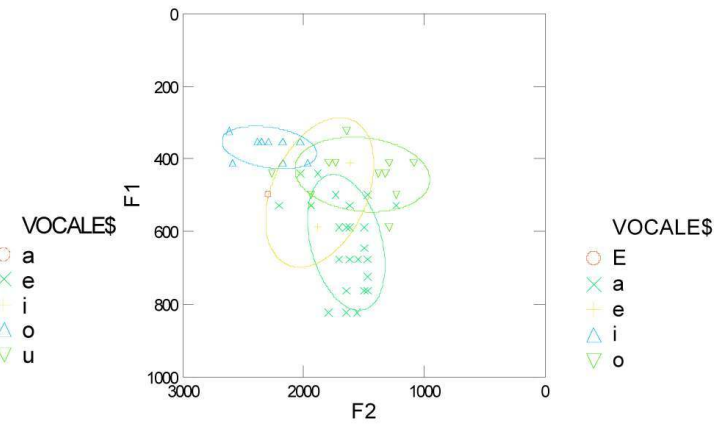

VOYELLES INDÉFINIES

Atones

HOMME 


\section{Figure 16}

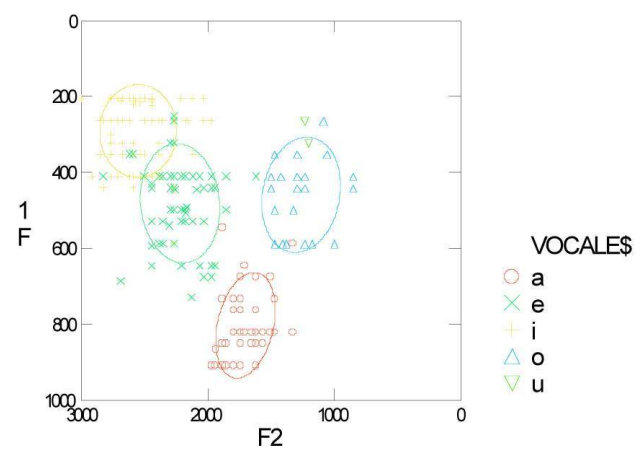

VOYELLES DÉFINIES VOYELLES INDÉFINIES

Atones

FEMME

Le sujet féminin présente le plus haut degré d'indéfinition dans les systèmes atones (final et non). L'analyse des les surfaces montre une tendence à l'hypoarticulation, qui ne se vérifie pas en position tonique, où les réalisations vocaliques se rapprochent plus largement aux attentes phonologiques.

\section{Figure 17}

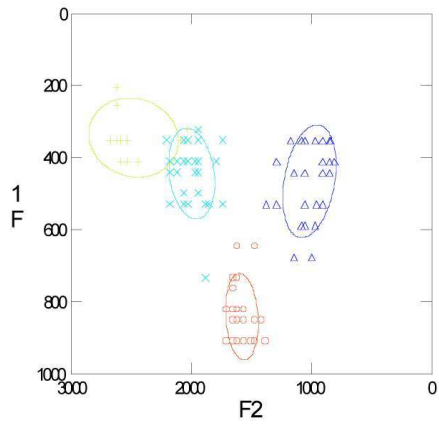

FINALES VRAIES

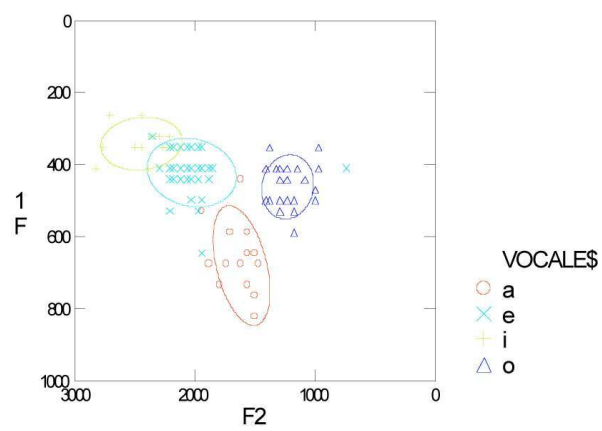

FINALES FAUSSES

Position : finales vraies/ fausses (parole dialogique) HOMME

\section{Figure 18}

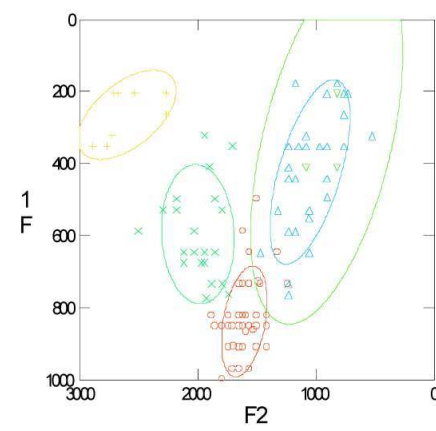

FINALES VRAIES

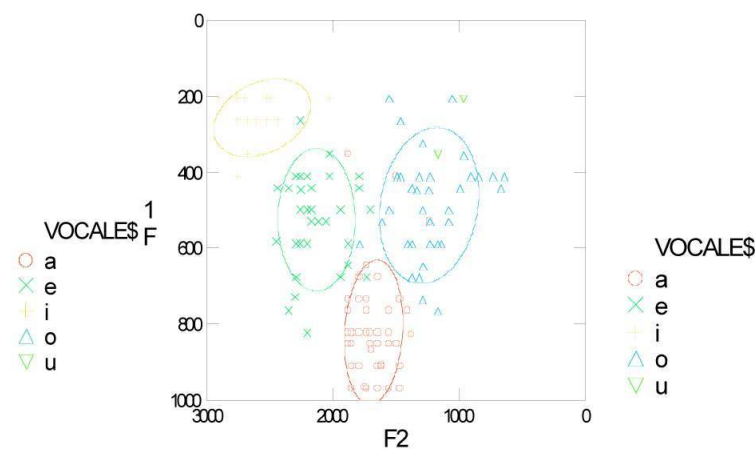

FINALES FAUSSES

Position : finales vraies/ fausses (parole dialogique) FEMME 
Les réalisations des deux locuteurs montrent une disposition commune: le degré de centralisation des finales fausses est plus large que dans les respectives systèmes toniques, atones et finales vraies. Il se présente en mesure plus importante dans la valeur de F2.

\section{Observations conclusives}

L'essai phonétique a montré :

- La difficulté à reconnaître, dans la chaîne phonique, des segments vocaliques avec traits acoustiques propres.

- La grande variabilité de toutes les réalisations, qui se montre par le déplacement continu de catégorie entre les timbres.

- La superposition des voyelles moyennes.

- Les fréquents phénomènes de réduction, en termes de centralisation et hypoarticulation.

- La plus haute tendance à la réduction des productions phoniques de la parole dialogique.

- La possibilité et nécessité de distinguer, dans le cadre des voyelles finales, des timbres avec réalisations différents.

À partir de ce résultat il faut s'interroger sur les mécanismes de perception linguistique, qui, de toute évidence, ne peuvent pas se baser sur un signal tellement déstructuré.

Ainsi les données expérimentales n'offrent pas seulement un cadre fidèle de la structure de la parole spontanée, mais favorisent aussi une vision plus complète de la communication et du langage en général.

\section{BIBLIOGRAPHIE}

Albano Leoni F., Caputo M.R., 1993. Vocalismo tonico e atono nel parlato italiano in Peretti A., Ferrero F. (a cura di) Atti del XXI Convegno Nazionale dell'Associazione Italiana di Acustica, Abbazia di Praglia (Padova) 31 marzo-2 aprile 1993, Padova, Arti grafiche padovane, pp. 75-80

Albano Leoni F., Caputo M.R., Cerrato L., Cutugno F., Maturi P., Savy R., 1994 Il vocalismo dell'italiano. Analisi di un campione televisivo in Perrone B. (a cura di) Atti del XXII Convegno Nazionale dell'Associazione Italiana di Acustica, Lecce 13-15 aprile 1994, Lecce, Cartografica Rosato, pp. 419-424

Calamai S. 2002 Vocali atone e toniche a Pisa in Regnicoli A. (a cura di) Atti delle XIII Giornate di studio del Gruppo di Fonetica Sperimentale - AIA, Macerata 13-15 dicembre 2001, Roma, Il Calamo, pp. 39-46.

CLIPS- Corpora e Lessici di Italiano Parlato e Scritto. Progetto n. 2 del Cluster C18 "LINGUISTICA COMPUTAZIONALE : RICERCHE MONOLINGUI E MULTILINGUI" (Legge 488), finanziato dal Ministero dell'Istruzione, dell'Università e della Ricerca (MIUR) (coordinatore nazionale : F. Albano Leoni). www.clips.unina.it 
Grimaldi M. 2003 Nuove ricerche sul vocalismo tonico del Salento meridionale. Analisi acustica e trattamento fonologico dei dati, Alessandria, Edizioni dell'Orso.

Lindblom B. 1963 Spectrographic study of vowel reduction in Journal of the Acoustic Society of America, vol. 35, pp. 1773-1781.

Lindblom B. 1990 Explaining phonetic variation: a sketch of the H\&H theory in Hardcastle W. J, Marchal A. (eds) Speech production and speech modelling, Kluwer Academic Press, Dodrecht, pp. 403-439.

Lo Prejato M., G. Clemente, R. Savy 2004 "Su alcuni aspetti della riduzione vocalica nella varietà napoletana", in A. De Dominicis et al. (acd), Costituzione, gestione e restauro di corpora vocali in Atti delle XIVe Giornate di Studio del GFS, Università della Tuscia (Viterbo), 4-6.XII.2003, Roma, Esagrafica : 183-188.

Moon S.-J., B. Lindblom 1994 "Interaction between Duration, Context, and Speaking Style in English Stressed Vowels", JASA, 96: 40-55.

Savy R., Cutugno F., 1997 Ipoarticolazione, riduzione vocalica, centralizzazione : come interagiscono nella variazione diafasica ? in Cutugno F. (a cura di) Atti delle VII Giornate del Gruppo di Fonetica Sperimentale - AIA, Napoli 14-15 novembre 1996, Roma, Esagrafica, pp. 177-194

van Bergem D. 1995 Acoustic and lexical vowel reduction, IFOTT, Amsterdam.

van Son R. 1993 Spectro-temporal features of vowel segments, Amsterdam, IFOTT.

\section{NOTES}

1. Albano Leoni et al., 1994; Savy \& Cutugno, 1997; Romito et al., 1997; Calamai, 2002; Lo Prejato et al., 2004.

2. Koopmans-van Beinum 1980; Harmegnies e Poch-Olivé 1992 ; van Son 1993 van Bergem 1995.

3. La version utilisée a été élaborée par l'Université de Napoli.

4. Grimaldi (2003) propose une analyse sur le vocalisme tonique relatif a la variété dialectal de Salentin méridional. Les données, pour la modalité d'acquisition et d'analyse, ne sont pas comparables. Toutefois, uniquement pour le vocalisme tonique de la parole lue prononcée par un locuteur masculin, une comparaison est possible et démontre un espace plus réduit pour la variété dialectale.

\section{RÉSUMÉS}

L'essai phonétique, une analyse acoustique sur les voyelles de la variété salentine de l'italien, se propose une recherche sur le phénomène de réduction vocalique, caractéristique propre de la parole spontanée qui se révèle selon des modalités différentes. La réduction est reliée au contexte phonique dans lequel les sons sont insérés, et aussi aux effets systématiques de la structure prosodique sur l'articulation des voyelles. On cherchera alors de reconnaître les 
différentes types de réduction, d'établir les différences entre eux et d'évaluer l'influence du contexte phonique sur eux.

The study, an acoustic analysis of Salentin Italian variety vowels,attempts a search on the vowel reduction phenomenon, a typical feature of speech who appears in different cases. The vowel reduction is tied to phonetic context, and also to systematic effects of prosodic structure on vowels articulation. We try to identify the different types of vowel reduction, and to establish the differences between them to verify the context influence.

INDEX

Mots-clés : réduction, cotexte, voyelles, parole spontanée, analyse acoustique

Keywords : reduction, cotext, vowels, connected speech, acoustic analysis

\section{AUTEUR}

ILARIA LIBERA MARGHERITA

Université de Pise, Italie 